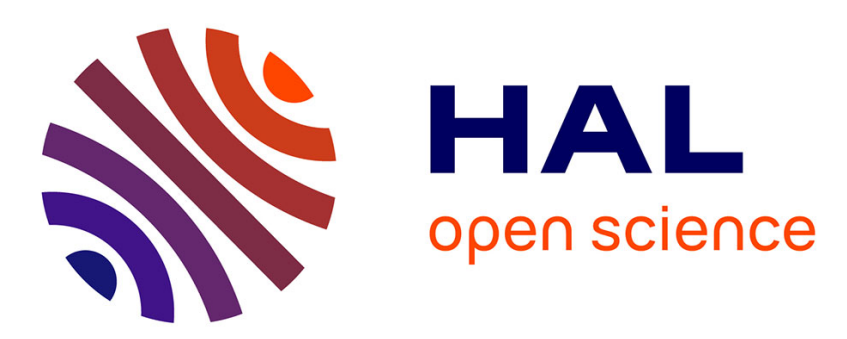

\title{
Light scattering by single ice crystals of cirrus clouds
}

Valery Shcherbakov, Jean-François Gayet, Olivier Jourdan, Johan Ström, Andreas Minikin

\section{To cite this version:}

Valery Shcherbakov, Jean-François Gayet, Olivier Jourdan, Johan Ström, Andreas Minikin. Light scattering by single ice crystals of cirrus clouds. Geophysical Research Letters, 2006, 33 (15), 10.1029/2006GL026055. hal-01823423

\section{HAL Id: hal-01823423 \\ https://hal.uca.fr/hal-01823423}

Submitted on 3 May 2021

HAL is a multi-disciplinary open access archive for the deposit and dissemination of scientific research documents, whether they are published or not. The documents may come from teaching and research institutions in France or abroad, or from public or private research centers.
L'archive ouverte pluridisciplinaire HAL, est destinée au dépôt et à la diffusion de documents scientifiques de niveau recherche, publiés ou non, émanant des établissements d'enseignement et de recherche français ou étrangers, des laboratoires publics ou privés. 


\title{
Light scattering by single ice crystals of cirrus clouds
}

\author{
Valery Shcherbakov, ${ }^{1,2}$ Jean-François Gayet, ${ }^{3}$ Olivier Jourdan, ${ }^{3}$ Johan Ström, ${ }^{4}$ \\ and Andreas Minikin ${ }^{5}$
}

Received 22 February 2006; revised 5 May 2006; accepted 14 June 2006; published 5 August 2006.

[1] In situ measurements of angular scattering intensities (ASIs) related to single ice crystals of cirrus clouds were obtained for the first time with the airborne Polar Nephelometer instrument. These observations were performed in the southern mid-latitudes during the INCA project. The analysis of the data shows that the well marked peaks of the ASIs are undoubtedly attributed to quasispecular reflections from crystal facets and the refracted rays passed through the ice crystals. Emphasizing that it is not enough to know the shape of the crystal, but also the internal structure is of great importance. Ray tracing simulations reproduce most of the observed ASIs features. The model of surface roughness, which assumes the Weibull statistics, enables to reproduce the quasi-specular external reflection from crystal facets. It follows from the retrievals that the surface of the crystals was deeply rough and the amount of the internal inclusions (air bubbles) was quite high. Citation: Shcherbakov, V., J.-F. Gayet, O. Jourdan, J. Ström, and A. Minikin (2006), Light scattering by single ice crystals of cirrus clouds, Geophys. Res. Lett., 33, L15809, doi:10.1029/2006GL026055.

\section{Introduction}

[2] Cirrus clouds are globally distributed with an annual global and local frequency of occurrence of about $30 \%$ [Wylie and Menzel, 1999]. They are composed almost exclusively of nonspherical ice crystals [e.g., Korolev et al., 1999; Gayet et al., 2004] and play an important role in the energy balance of the earth-atmosphere system through their interactions with solar and terrestrial radiation [Liou, 1992]. The accurate modeling of cirrus single-scattering parameters is the prior condition for the interpretation of remote-sensing measurements [C.-Labonnote et al., 2000]. There were developed parameterizations of scattering and geometric properties of a variety of ice crystal shapes and sizes for shortwave bands [Yang et al., 2000]. Theoretical and numerical techniques, laboratory measurements, and practical applications of light scattering by nonspherical particles were reviewed in the book edited by Mishchenko et

\footnotetext{
${ }^{1}$ Institute of Physics, Minsk, Belarus.

${ }^{2}$ Also at Observatoire de Physique du Globe de Clermont-Ferrand (OPGC), Laboratoire de Météorologie Physique, UMR/CNRS 6016, Université Blaise Pascal, Clermont-Ferrand, France.

${ }^{3}$ Laboratoire de Météorologie Physique UMR 6016 CNRS, UniversitéBlaise Pascal, Aubière, France.

${ }^{4}$ Department of Applied Environmental Science, Stockholm University, Stockholm, Sweden.

${ }^{5}$ Institut für Physik der Atmosphäre, DLR, Oberpfaffenhofen, Wessling, Germany.
}

Copyright 2006 by the American Geophysical Union. 0094-8276/06/2006GL026055 al. [2000]. Despite the substantial progress in this domain, the optical characteristics of ice crystals measured in natural conditions are still needed, first of all for validation of numerical techniques and the determination of free parameters of models.

[3] Examples of angular scattering intensities (ASIs) of single ice crystals were already reported in the work Shcherbakov et al. [2006] (hereinafter referred to S06). The data were obtained from ground-based observations at the Amundsen-Scott South Pole Station during an episode of diamond dust.

[4] This paper presents typical angular scattering intensities of single ice crystals measured for the first time in cirrus clouds and discusses the modeling outcomes.

\section{Experimental Conditions, Instrumentation, and Data Processing}

[5] Airborne measurements of optical and microphysical properties of mid-latitude cirrus clouds were carried out in the Southern hemisphere from Punta Arenas $\left(53^{\circ} \mathrm{S}, 71^{\circ} \mathrm{W}\right)$ between March 23 and April 13, 2000 during the INCA experiment (Interhemispheric differences in cirrus properties from anthropogenic emissions) [Ström et al., 2001]. The instruments operated onboard the German DLR (Deutsches Zentrum für Luft- und Raumfahrt) Falcon aircraft (see details in [Gayet et al., 2004]), included the Polar Nephelometer (PN) [Gayet et al., 1997]. This instrument measures the angular scattering intensities at scattering angles from about $5^{\circ}$ to $169^{\circ}$. The reliability of the data and the uncertainties of the derived optical parameters were discussed in detail by Auriol et al. [2001] and Gayet et al. [2004]. The method of data processing used in this study was described in details in [SO6]. We recall that the PN measures ASIs as a two-dimensional scattering pattern defined by the scattering angle $\theta$ and by the azimuthal angle $\varphi$. The latter variable takes only one of two values, $0^{\circ}$ or $180^{\circ}$ [see $S 06$, Figure 2]. If the acquisition frequency is high and the concentration of particles is low enough, the PN records ASIs of single crystals. Generally, in such a case the ASIs depend on $\varphi$ and the orientation of a particle. The notations "upward" and "downward" angles and/or ASIs will be used to indicate two values of the azimuthal angle $\varphi$, that is, $0^{\circ}$ and $180^{\circ}$. The available channels document ASIs at 26 upward and 9 downward scattering angles. Moreover, the Polar Nephelometer provides only ASIs, that is, the ice particles properties (size, shape, and others) are not directly observed and have to be retrieved.

[6] During the INCA experiment, there were recorded several time series at the frequency of $100 \mathrm{~Hz}$. In such a case and at the aircraft cruise speed of $170 \mathrm{~m} / \mathrm{s}$, the PN sampling volume is close to $85 \mathrm{~cm}^{3}$. Accordingly, the ice particle concentration has to be lower than approximately 
$10 \mathrm{~L}^{-1}$ if it is expected to record ASIs of no more than one ice particle per strobe. That condition can be violated inside cirrus even for particles having an effective diameter larger than $100 \mu \mathrm{m}$ that typically are few in number [Gayet et al., 2004]. In order to reduce (i) the probability of coincidence of two particles at one strobe, and (ii) the effect of concurrence of small particles in the sampling volume, there were considered for further analysis only those time series that were recorded at cloud edges where the ice particle concentration was low. In addition, there were selected only those ASIs that belong to time periods where each occurrence of the signal from an ice crystal in the PN time series was surrounded by several strobes of the background signal. Accordingly, we deal with rather specific conditions in this study, and whether the results can be considered as the representative for cirrus clouds in general is open to question.

[7] The data discussed below were collected on March 24 and March 31 at flight levels of $-30^{\circ} \mathrm{C} / 6200 \mathrm{~m} / 447 \mathrm{hPa}$ and $-45^{\circ} \mathrm{C} / 9200 \mathrm{~m} / 301 \mathrm{hPa}$, respectively, and with relative humidity (with respect to ice) ranged from $100 \%$ to $130 \%$ in both cases. In total, there were obtained over 47000 ASIs of single ice crystals in cirrus clouds. In over 600 cases, the upward and downward ASIs are certainly different. The well marked local maximum (or maxima) are usually present and can be undoubtedly attributed to quasi-specular reflections from crystal facets or the refracted rays passed through the ice crystals. We note in passing that the term "quasi-specular" is used to describe a reflection from a rough surface, which differs from the perfect plane specular reflection, that is, the delta-like peak of the intensity. The reflections from facets and the refracted rays are the reason for high variability of the recorded ASIs. For instance, normalized ASIs vary about two orders of magnitude at sideward angles. The variability essentially decreases when an ensemble of randomly orientated particles is dealt with [S06].

\section{Modeling Approach}

[8] The approach used to retrieve ice particle parameters and model the measured ASIs was described in detail in [S06]. Briefly, it consists in the following.

[9] The crystal is assumed to be a hexagonal column/ plate. The Monte Carlo ray tracing method Macke et al. [1996a, 1996b] was applied to compute angular scattering intensities. The code was adapted to the PN optical scheme (including the optical aperture of the receivers), that is, to provide $2 \mathrm{D}$ scattering patterns of a single ice crystal having specified orientations in the sensitive volume. Moreover, a new model of surface roughness based on the Weibull statistics [e.g., Dodson, 1994] was proposed in [S06]. Accordingly, the probability distribution function of tiltangles is specified by two parameters: the scale parameter $\sigma$ and the shape parameter $\eta$ (which determines the kurtosis).

[10] The modified Monte Carlo ray tracing code was used to generate a lookup table of the single ice crystal ASIs. The input parameters are: the three Euler angles, which determine the orientation of the particle, the aspect ratio of the ice crystal, the two parameters of the surface roughness, and the mean free path length (MFPL) between two subsequent scattering (on inclusions) events. The last parameter is to model air bubbles that may be trapped inside ice particles [Macke et al., 1996b]. It may be considered as some 'equivalent-morphological' model of internal density variations. The ASIs were calculated assuming: (i) the incident wavelength of $0.8 \mu \mathrm{m}$, and (ii) the complex refractive index for ice tabulated by Warren [1984].

[11] The lookup table was used to fit the measured ASIs (at all 35 angles simultaneously) and then to retrieve the corresponding parameters of single ice crystals. It should be stressed that an assessment of characteristics of a particle from the measured ASIs is an extremely challenging task. This is not only due to the high number of the dimensions of the lookup table, but also to the essential nonlinearity of the relationship between the particle parameters and the ASIs. The method and retrieval uncertainties have been addressed in [S06]. The ASIs of a single crystal are more informative than a phase function of an ensemble of particles. Such a number of parameters can be retrieved because they chiefly affect distinct attributes of ASIs. For instance, the Euler angles specify the position of the quasi-specular reflection maximum on the $\theta$-scale; the width and the sharpness of that maximum basically depend on the scale parameter $\sigma$ and the shape parameter $\eta$, respectively; internal inclusions mainly influence the range of ASIs.

\section{Representative Examples}

[12] The collection of the ASIs that depend on the azimuthal angle $\varphi$ (over 600 cases) was selected for the modeling and the crystal parameters retrievals. A representative example of ASIs measured on March 24 at 21:29:40 UTC is shown in Figure 1a. The upward and downward measurements are plotted with full circles and open triangles, respectively. The ASIs of Figure 1a highlight a very well marked upward peak centered at $\theta=71^{\circ}$ with a magnitude even higher than the scattering intensity at $\theta=9^{\circ}$. Although 9 downward angles are available (against 26 upward angles), Figure 1a shows that the peak value at $109.5^{\circ}$ is about 2.2 times higher than the downward ASIs value at $39.5^{\circ}$. The retrieved parameters of the ice crystal are listed in Table 1 (case A). We note in passing that the uncertainties in Table 1 correspond to propagation of measurement errors [e.g., Bevington, 1969]. The aspect ratio is 1 (solid column), the roughness parameters $\sigma$ and $\eta$ are 0.09 and 0.75 , respectively. The estimated size parameter $(\mathrm{D}=$ $74 \mu \mathrm{m})$ of the ice crystal is also reported in Table $1 . D$ is the diameter of the circle circumscribing the hexagonal facet of the ice crystal. Table 1 reports the MFPL ratio, which is equal to the mean free path length divided by the parameter $D$. The ratio is of 0.5 , that is, rather low. This implies that a light ray passing through the crystal hits an air bubble on overage twice, and, the effect of air bubbles on the ASIs is quite high.

[13] Figure 1a also superimposes the model results (black and gray curves for the upward and downward angles, respectively). The agreement is good enough to reproduce most of the characteristic features of the observed ASIs for both upward and downward angles. From the analysis of the 2D scattering patterns of the corresponding pristine crystal, the $71^{\circ}$ and $109.5^{\circ}$ peaks correspond to the quasi-specular reflection from the side and the hexagonal facets, respectively. The downward local maximum at $42^{\circ}$ is due to the 

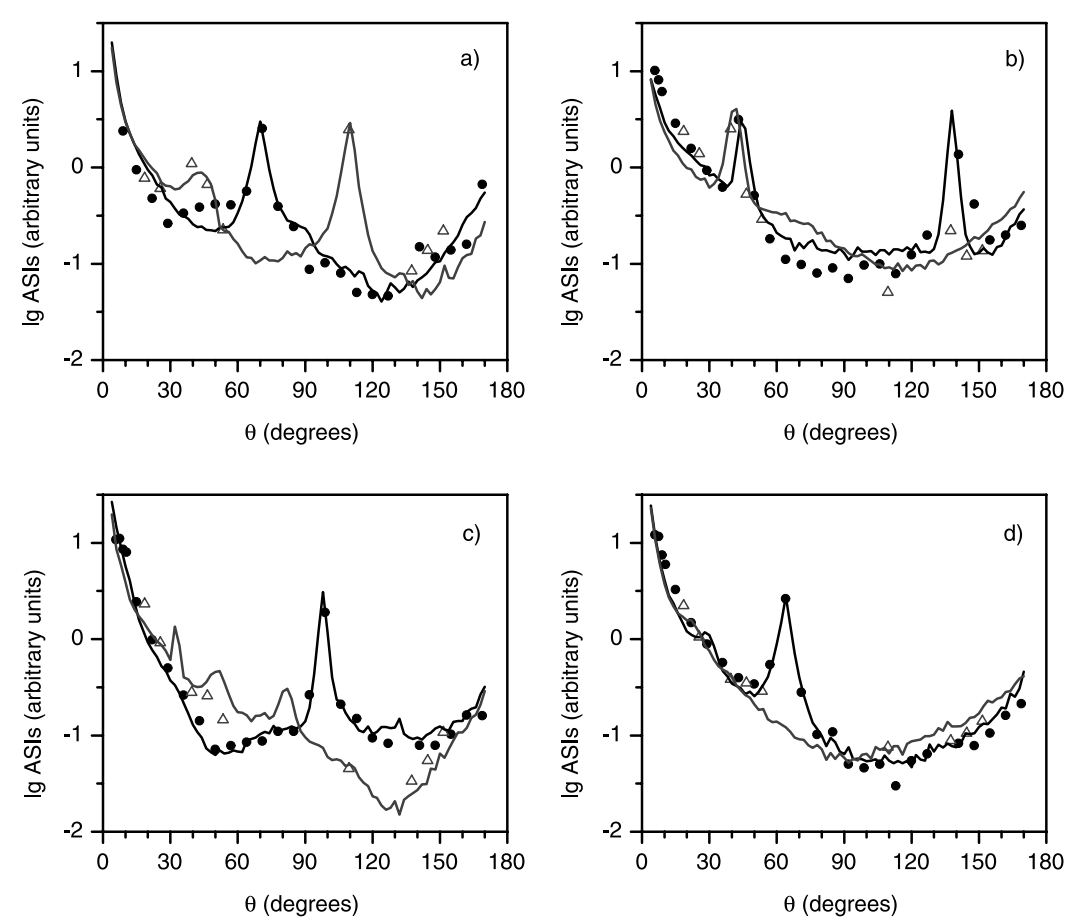

Figure 1. $(\mathrm{a}-\mathrm{d})$ Angular scattering intensities measured by the Polar Nephelometer (solid circles and open triangles are the upward and downward values, respectively). ASIs modeled by the ray tracing technique (upward and downward components are represented with black and gray curves, respectively). Figures $1 \mathrm{a}-1 \mathrm{~d}$ correspond to cases A-D in Table 1 .

refracted rays. According to the orientation of the ice crystal (see the Euler angles values in Table 1), the peaks of the reflections were within the field-of-view of the receiving photodiodes. At the same time, Figure 1a reveals significant differences between the observed and model ASIs. These differences are much larger than the measurement error estimates and cannot be attributed to instrument shortcomings.

[14] Figure 1b shows an example (the symbols are the same as in Figure 1a) when the 2D scattering patterns correspond to a hexagonal plate. The estimated value of the aspect ratio is of 0.5 (see Table 1 , case B). The ice crystal was sampled on March 24 at 21:41:34 UTC. The local maxima at $138^{\circ}$ upward and at $42^{\circ}$ downward angles are due to the quasi-specular external reflections from the side and hexagonal facets, respectively. The peak at $44^{\circ}$ is formed by the refracted rays. In general, the model follows well enough the main features of the experimental data. On the other hand, the discrepancies at the sideward scattering angles exceed the measurement errors.

[15] The agreement between the observed and model ASIs shown in Figure $1 \mathrm{c}$ is almost perfect. The measured downward ASIs even have some indication of the $52^{\circ}$ maximum. The last one is formed by rays having internal reflections. The ASIs were recorded on March 31 at 21:12:47 UTC. According to the retrieval (see Table 1, case $\mathrm{C}$ ), the crystal was an elongated column having a significant amount of internal inclusions (air bubbles).

[16] A representative example of the ASIs having only one local maximum is shown in Figure 1d. The ice crystal was sampled on March 31 at 21:40:22 UTC. The upward peak is due to the external reflection from the side facet of the hexagonal plate (see Table 1, case D). According to the retrieved orientation of the particle, other refraction and reflection peaks passed outside the $\mathrm{PN}$ receivers.

Table 1. Retrieved Values of the Ice Crystals Parameters Related to Four Examples of Measurements ${ }^{\mathrm{a}}$

\begin{tabular}{lcccc}
\hline Parameter & Case A & Case B & Case C & Case D \\
\hline$\alpha^{\circ}$ & $89 \pm 1$ & $85 \pm 1$ & $86 \pm 1$ & $16 \pm 1$ \\
$\beta^{\circ}$ & $145 \pm 1$ & $111 \pm 1$ & $131 \pm 1$ & $144 \pm 1$ \\
$\gamma^{\circ}$ & $30 \pm 2$ & $28 \pm 1$ & $27 \pm 2$ & $35 \pm 1$ \\
Aspect ratio & $1 \pm 0.25$ & $0.5 \pm 0.2$ & $3 \pm 0.5$ & $0.5 \pm 0.2$ \\
$\eta$ & $0.75 \pm 0.02$ & $0.75 \pm 0.02$ & $0.75 \pm 0.02$ & $0.75 \pm 0.02$ \\
$\sigma$ & $0.09 \pm 0.01$ & $0.05 \pm 0.01$ & $0.05 \pm 0.01$ & $0.1 \pm 0.01$ \\
MFPL ratio & $0.5 \pm 0.1$ & $0.125 \pm 0.005$ & $0.38 \pm 0.05$ & $0.38 \pm 0.05$ \\
$D, \mu \mathrm{m}$ & 74 & 122 & 78 & 151 \\
\hline
\end{tabular}

${ }^{\mathrm{a}}$ The three Euler angles $\alpha, \beta$, and $\gamma$ which define of the particle orientation; the aspect ratio; the surface roughness parameters $\eta$ and $\sigma$; the mean free path length (MFPL) ratio and the diameter $D$ of the circle circumscribing the hexagonal facet of an ice crystal. 
[17] According to images of the PMS 2D-C probe [Gayet et al., 2002], some ice columns and plates were observed at the sampling periods analysed above. At the same time, irregular polycrystals dominated the particle population of the 24 March cirrus (cases A \& B, see Table 1) and bulletrosettes prevailed during the 31 March cirrus (cases C \& D).

\section{Discussion}

[18] Generally, the observed angular scattering intensities in cirrus clouds resemble those one that were recorded during the episode of diamond dust [SO6]. This is interesting as this may suggest similar formation and growth processes.

[19] It follows from the modeling of over 600 ASIs that the retrieved values of the parameter $\eta$ were always between 0.74 and 0.76 , that is, the peaks of quasi-specular reflection are much sharper than it was used by Yang and Liou [1998]. The parameter $\sigma$ was within the range of $0.05-0.2$, which corresponds to deeply rough surface according to the classification of Yang and Liou [1998]. The same features were observed in the diamond dust case [SO6]. In contrast, the retrieved values of the MFPL ratio are much lower in the cirrus particles case. In other words, the effect of the internal inclusions (air bubbles) is higher in high-altitude clouds, and consequently the range of ASIs variation is lower (ratio of about 1.6, on average). These findings support the results of the work of Knap et al. [2005], who achieved the best simulation of remote sensing data for models of ice clouds consisting of crystals with distorted surfaces or containing air bubbles.

[20] Comparing to the case of pure hexagonal crystals, both contaminating factors such as surface roughness or the amount of the inclusions smooth the phase function of an ensemble of randomly oriented particles [e.g., Macke et al., 1996b; Yang and Liou, 1998]. And, the air bubbles inclusions generally decrease the asymmetry parameter $g$ [Macke et al. 1996b]. At the kurtosis parameter $\eta$ of 0.75 , the $22^{\circ}$ and $46^{\circ}$ halo peaks vanish at the roughness scale parameter $\sigma$ of about 0.2 without inclusions, and of about 0.1 when the MFPL ratio is of 0.5 . (The wavelength is of $0.8 \mu \mathrm{m}$ and the particle dimensions are of Table 1.) The value of $g$ decreases within $4 \%$ when $\sigma$ increases to 0.2 . At the same time, the asymmetry parameter could be reduced up to $20 \%$ due to the internal inclusions (at the MFPL ratio of 0.125 ).

[21] Concerning the quality of the ASIs fitting, the model, as a rule, follows quite well the main features. Especially, the maxima of the quasi-specular external reflection and their wings are usually very well fitted. At the same time, we have not succeeded to achieve a flawless agreement. (The modeldata agreement like that one shown in Figure 1c is rare.) The difference may be partially due to a crystal deviation from the ideal hexagonal shape. On the other hand, the majority of the discrepancies are, in our opinion, caused by neglected features of rays/electromagnetic waves spreading in the interior of an ice crystal. For instance, it can be frequently observed from CPI images that particles are not homogeneous, nor symmetric. There are internal structures, which look like variations of the density [e.g., Lawson et al., 2006]. The subsequent variations of the refractive index may significantly affect directions of propagation of rays passing through the crystal.
[22] Another point is that, even when the particle surface is very smooth and has only a few elements, one needs very large size parameters in order to reduce considerably the geometrical optics errors [Mishchenko and Macke, 1999]. It would be better to model ASIs using an algorithm strictly based on the electromagnetic theory and, in particular, include explicitly the effects of diffraction on individual surface elements (micro-facets) which tend to spread the scattered light around the specular reflection or the refraction direction [Mishchenko et al., 1997].

[23] In addition, the discrepancies mentioned above may be partially due to the "near field" interactions inside a crystal, especially when the amount of the internal inclusions is high [Mishchenko et al., 1995]. The contribution of the "near field" interactions, when the distance between inclusions and/or between the surface and inclusions is not large enough, should also be investigated on the base of the electromagnetic theory.

\section{Conclusions}

[24] The representative examples of the unique data on angular scattering intensities of single ice crystals of cirrus clouds are presented. The range of ASIs is large due to quasi-specular reflections from crystal facets or refracted rays.

[25] The ray tracing technique is validated by modeling ASIs of large crystals. The model, as a rule, follows quite well the main features. Especially, the maxima of the quasispecular external reflection and their wings are usually very well fitted. The discrepancies between the model and the recorded data are supposed to be caused by neglected features of rays/electromagnetic waves spreading in the interior of an ice crystal.

[26] The model free parameters were estimated. The surface of the cirrus crystals was deeply rough and the amount of the internal inclusions (air bubbles) was quite high. That perceptibly decreases the asymmetry parameter.

[27] Acknowledgments. Thanks are due to our INCA colleagues for their helpful contribution to the experiments. We are grateful to the members of the DLR who operated the Falcon aircraft during the experiments. The authors are grateful to A. Macke for providing his Monte Carlo ray tracing code. This work was funded by a grant from the CNRS/PATOM committee.

\section{References}

Auriol, F., J.-F. Gayet, G. Febvre, O. Jourdan, L. Labonnotte, and G. Brogniez (2001), In situ observations of cirrus cloud scattering phase function with $22^{\circ}$ and $46^{\circ}$ halos: Cloud field study on 19 February 1998 , J. Atmos. Sci., 58, 3376-3390.

Bevington, P. R. (1969), Data Reduction and Error Analysis for the Physical Sciences, pp. 58-64, McGraw-Hill, New York.

C.-Labonnote, L., G. Brogniez, M. Doutriaux-Boucher, J. Buriez, J. Gayet, and H. Chepfer (2000), Modeling of light scattering in cirrus clouds with inhomogeneous hexagonal monocrystals. Comparison with in-situ and ADEOS-POLDER measurements, Geophys. Res. Lett., 27(1), 113-116. Dodson, B. (1994), Weibull Analysis, 256 pp., ASQC Quality Press, Milwaukee, Wisconsin.

Gayet, J.-F., O. Crepel, J.-F. Fournol, and S. Oshchepkov (1997), A new airborne polar Nephelometer for the measurements of optical and microphysical cloud properties. part I: Theoretical design, Ann. Geophys., 15(4), 451-459.

Gayet, J.-F., F. Auriol, A. Minikin, J. Ström, M. Seifert, R. Krejci, A. Petzold, G. Febvre, and U. Schumann (2002), Quantitative measurement of the microphysical and optical properties of cirrus clouds with four different in situ probes: Evidence of small ice crystals, Geophys. Res. Lett., 29(24), 2230, doi:10.1029/2001GL014342. 
Gayet, J., J. Ovarlez, V. Shcherbakov, J. Ström, U. Schumann, A. Minikin, F. Auriol, A. Petzold, and M. Monier (2004), Cirrus cloud microphysical and optical properties at southern and northern midlatitudes during the INCA experiment, J. Geophys. Res., 109, D20206, doi:10.1029/ 2004JD004803.

Knap, W. H., L. C-Labonnote, G. Brogniez, and P. Stammes (2005), Modeling total and polarized reflectances of ice clouds: Evaluation by means of POLDER and ATSR-2 measurements, Appl. Opt., 44, 4060-4073.

Korolev, A. V., G. A. Isaac, and J. Hallett (1999), Ice particle habits in Arctic clouds, Geophys. Res. Lett., 26(9), 1299-1302.

Lawson, R. P., B. A. Baker, B. Pilson, and Q. Mo (2006), In Situ observations of the microphysical properties of wave, cirrus and anvil clouds. part II: Cirrus Clouds, J. Atmos. Sci, in press..

Liou, K. N. (1992), Radiation and Cloud Processes in the Atmosphere, 487 pp., Oxford Univ. Press, New York.

Macke, A., J. Mueller, and E. Raschke (1996a), Single scattering properties of atmospheric ice crystals, J. Atmos. Sci., 53, 2813-2825.

Macke, A., M. I. Mishchenko, and B. Cairns (1996b), The influence of inclusions on light scattering by large ice particles, J. Geophys. Res., 101(D18), 23,311-23,316.

Mishchenko, M. I., and A. Macke (1999), How big should hexagonal ice crystals be to produce halos?, Appl. Opt., 38, 1626-1629.

Mishchenko, M. I., D. W. Mackowski, and L. D. Travis (1995), Scattering of light by bispheres with touching and separated components, Appl. Opt., 34, 4589-4599.

Mishchenko, M. I., D. J. Wielaard, and B. E. Carlson (1997), T-matrix computations of zenith-enhanced lidar backscatter from horizontally oriented ice plates, Geophys. Res. Lett., 24(7), 771-774.
Mishchenko, M. I., J. W. Hovenier, and L. D. Travis (Eds.) (2000), Light Scattering by Nonspherical Particles: Theory, Measurements, and Applications, 690 pp., Elsevier, New York.

Shcherbakov, V., J.-F. Gayet, B. A. Baker, and R. P. Lawson (2006), Light scattering by single natural ice crystals, J. Atmos. Sci., 63, 1513-1525.

Ström, J., et al. (2001), Aerosol and cirrus measurements at midlatitudes on the Southern hemisphere: An overview based on the first INCA experiment, Rep. EUR 19,428 EN, Eur. Comm., Brussels, Belgium.

Warren, S. G. (1984), Optical constants of ice from the ultraviolet to the microwave, Appl. Opt., 23, 1206-1225.

Wylie, D. P., and W. P. Menzel (1999), Eight years of global high cloud statistics using HIRS, J. Clim., 12, 170-184.

Yang, P., and K. N. Liou (1998), Single-scattering properties of complex ice crystals in terrestrial atmosphere, Contrib. Atmos. Phys., 71, 223-248.

Yang, P., K. N. Liou, K. Wyser, and D. Mitchell (2000), Parameterization of the scattering and absorption properties of individual ice crystals, J. Geophys. Res., 105, 4699-4718.

J.-F. Gayet and O. Jourdan, Laboratoire de Météorologie Physique UMR 6016 CNRS, Université Blaise Pascal, 24, Avenue des Landais, F-63177 Aubière, France. (gayet@opgc.univ-bpclermont.fr)

A. Minikin, Institut für Physik der Atmosphäre, DLR, Oberpfaffenhofen, P.O. Box 1116, D-82230 Wessling, Germany.

V. Shcherbakov, Institute of Physics, 66 Independence Avenue, Minsk BY-220072, Belarus.

J. Ström, Department of Applied Environmental Science, Stockholm University, Frescativägen 54 a, SE-10691 Stockholm, Sweden. 